\title{
Clinical comparison between conventional and microdissection testicular sperm extraction for non-obstructive azoospermia: Understanding which treatment works for which patient
}

\author{
Elia Maglia, Luca Boeri, Matteo Fontana, Andrea Gallioli, Elisa De Lorenzis, Franco Palmisano, \\ Stefano Paolo Zanetti, Gianluca Sampogna, Liliana Restelli, Edgardo Somigliana, Mariapia Serrago, \\ Franco Gadda, Emanuele Montanari
}

IRCCS Fondazione Ca' Granda, Ospedale Maggiore Policlinico, Department of Urology, Milan, Italy.

\section{Summary Objectives: The superiority of microdissec- tion testicular sperm extraction (mTESE)} over conventional TESE (cTESE) for men with non-obstructive azoospermia (NOA) is debated. We aimed to compare the sperm retrieval rate (SRR) of mTESE to cTESE and to identify candidates who would most benefit from mTESE in a cohort of Caucasian-European men with primary couple's infertility. Material and methods: Data from 49 mTESE and 96 cTESE patients were analysed. We collected demographic and clinical data, serum levels of LH, FSH and total testosterone. Patients with abnormal karyotyping were excluded from analysis. Age was categorized according to the median value of 35 years. FSH values were dichotomized according to multiples of the normal range $(\mathrm{N})$ (N and 1.5 N: 1-18 mIU/mL, and > 18 $\mathrm{mIU} / \mathrm{mL}$ ). Testicular histology was recorded for each patient. Descriptive statistics and logistic regression analyses tested the impact of potential predictors on positive SRR in both groups. Results: No differences were found between groups in terms of clinical and hormonal parameters with the exception of FSH values that were higher in mTESE patients $(p=0.004)$. SRR were comparable between mTESE and cTESE (49.0\% vs. $41.7 \%, p=0.40)$. SRRs were significantly higher after mTESE in patients with Sertoli cell-only syndrome (SCOS) $(p=0.038)$, in those older than 35 years $(p=0.03)$ and with $\mathrm{FSH}>1.5 \mathrm{~N}$ $(p<0.001)$, as compared to men submitted to cTESE. Multivariable logistic regression analysis showed that mTESE was independent predictor of positive SR in patients older than 35 years $(p=0.002)$ and with FSH $>1.5 \mathrm{~N}(p=0.018)$.

Moreover, increased FSH levels $(p=0.03)$ and both SCOS $(p=0.01)$ and MA histology $(p=0.04)$ were independent predictors of SRR failure.

Conclusions: Microdissection and cTESE showed comparable success rates in our cohort of patients with NOA. mTESE seems beneficial for patients older than 35 years, with high FSH values, or when SCOS can be predicted. Given the high costs associated with the mTESE approach, the identification of candidates most likely to benefit from this procedure is a major clinical need.

KEY WORDS: Testicular sperm extraction; Non-obstructive azoospermia; Infertility; Risk factors.

Submitted 17 February 2018; Accepted 10 April 2018

\section{INTRODUCTION}

In contrast to obstructive azoospermia in which there is an obstruction in the ductal system, non-obstructive azoospermia (NOA) refers to the absence of spermatozoa in semen analysis due to minimal or no production of fully developed spermatozoa in the testicles.

Approximately 1\% of all men and 10\% of infertile men are affected by testicular failure as a result of NOA (1). Testicular spermatozoa can be retrieved in some NOA men even despite the absence of ejaculated spermatozoa in their semen, from isolated foci of active spermatogenesis. The chance of fatherhood for the NOA patient has changed dramatically in recent years following the introduction of surgical sperm retrieval techniques, in particular conventional TESE (cTESE) and microdissection TESE (mTESE). Until recently, cTESE represented the first-line option to retrieve spermatozoa in NOA subjects for intracytoplasmic sperm injection (ICSI) (2). However, since its introduction in 1999, mTESE has continuously been found to be associated with better sperm retrieval rates (SRRs) and fewer complications compared to cTESE (3). Direct vision with the operating microscope in MTESE is of great advantage as larger, whitish tubules, presumably containing more germ cells with active spermatogenesis, can be identified. Indeed, as reported by various authors, the rate of positive SRR differs significantly between the two techniques, ranging from about $63 \%$ to $42 \%$ in mTESE and from $16 \%$ to $45 \%$ in cTESE (4-6). Moreover, cTESE can be undermined by potential surgical complications, which are virtually absent in the mTESE series $(7,8)$. This should not be ignored given that a significant number of azoospermic patients are typically affected by secondary disturbances, such hypogonadism, for which mTESE may allow for better preservation of the testicular tissue. On the contrary, other authors have failed to find a significant superiority of mTESE over CTESE in terms of SRRs $(9,10)$. Moreover, the overall positive SRR, lower costs and high reproducibility of cTESE represent unique characteristics that should be taken into account when counseling NOA infertile couples (10). Microdissection TESE, indeed, is a technically challeng- 
ing and expensive procedure that requires dedicated equipment and specifically trained surgeons.

Considering the different characteristic of each surgical technique and the high heterogeneity in the current literature regarding which technique is superior, we believe it to be of paramount importance to define which patients could benefit most from a complex surgery such as microdissectional TESE. The aim of the present study is to compare SRR by mTESE with that obtained by conventional TESE in NOA patients and to identify the patients who could most benefit from a microsurgical approach by finding the best predictors of positive sperm retrieval in our cohort.

\section{MATERIALS AND MEtHOdS}

The analyses of this cross-sectional study were based on a sample of 145 consecutive white-European men assessed at a single academic centre for primary couple's infertility (non-interracial infertile couples only) between January 2012 and April 2017. The initial cohort included 149 patients, however 4 (2.68\%) men were excluded from the final analysis for missing values. According to the World Health Organisation (WHO) criteria, infertility was defined as not conceiving a pregnancy after at least 12 months of unprotected intercourse regardless of whether or not a pregnancy ultimately occurred (11). Primary infertility is defined as when a couple has never been able to conceive (11). Infertile patients were enrolled if they were between 18 and 55 years of age and had only male factor infertility (MFI); MFI was defined after a comprehensive gynecological evaluation of the female partner. All patients were diagnosed with NOA on the basis of a complete history, physical examination, endocrine profile, and chromosomal analysis before being scheduled for TESE with sperm freezing.

All patients underwent chromosomal analysis and those with abnormal karyotyping were excluded from the study cohort. Patients underwent at least two consecutive semen analyses, both showing absence of spermatozoa in the ejaculate after centrifugation. Semen samples were collected by masturbation and analysed within $2 \mathrm{~h}$ according to the WHO criteria.

Patients were assessed with a thorough medical history including age and comorbidities. Age was categorized according to the median value of 35 yrs. Comorbidities were scored with the Charlson Comorbidity Index (CCI) (12). We used the International Classification of Diseases, $9^{\text {th }}$ revision. For the specific purpose of the analysis, CCI was categorized as 0 or $\geq 1$. All individuals were sexually active, reporting to have intercourse at least four times per month. Body mass index (BMI) defined as weight in kilograms by height in square meters, was calculated. BMI was considered using the cut offs proposed by the National Institutes of Health (NIH): normal weight (18.5-24.9), overweight (25.0-29.9), and class $\geq 1$ obesity ( $\geq 30.0$ ). Lifestyle factors potentially related with any impairment of semen quality were carefully assessed. Colour-Doppler ultrasound was used to detect spermatic vein reflux and to classify the grade of varicocele in infertile patients. As a main entry criterion for the study, only patients with complete data collection were included; therefore, patients with incomplete medical history were excluded. Venous blood samples were drawn from each infertile patient between $7 \mathrm{AM}$ and 11 AM after an overnight fast. Follicle-stimulating hormone (FSH), luteinising hormone (LH) and prolactin (PRL) were measured using a heterogeneous competitive magnetic separation assay (Bayer Immuno 1 System, Bayer Corp., Tarrytown, NY, USA). Total testosterone (tT) levels were measured via a direct chemiluminescence immunoassay (ADVIA Centaur; Siemens Medical Solutions Diagnostics, Deerfield, IL, USA). Hypogonadism was defined as tT less than $3 \mathrm{ng} / \mathrm{ml}$ (13). The same laboratory analyzed all parameters for all patients. FSH values were categorized into two groups according to multiples of the normal range $(\mathrm{N})(\mathrm{N}$ and 1.5N: $1-18 \mathrm{mIU} / \mathrm{mL}$, and $>18 \mathrm{mIU} / \mathrm{mL}$ ).

Patients included in the study were scheduled for conventional cTESE or mTESE based on the availability of the surgical waiting list. Informed consent was obtained after a thorough explanation of results in the literature and the invasiveness of the surgical technique. Testicular histology was recorded based on the predominant histological pattern, such as hypospermatogenesis (HS), maturation arrest (MA) and Sertoli cell-only syndrome (SCOS). The same pathologist reviewed all the tissue samples.

Conventional TESE was performed, under general or local anesthesia, unilaterally on the larger testis; when testes volume was equal, the right testis was used. Through a small horizontal incision in the right-median part of the scrotal, the skin, dartos muscle, and tunica vaginalis were opened to expose the tunica albuginea. The tunica albuginea was incised for about $5 \mathrm{~mm}$ at the middle of the testis. Multiple testicular specimens were excised and dispersed between two glass slides, and the embryologist observed the samples under the optic microscope. If no sperm were seen in the initial sample, subsequent samples were taken from other locations, in the upper and lower pole of the testis, and subsequently from the contralateral testis (in only $12 \%$ of the cases). Microdissection TESE was performed under general anesthesia according to the procedure reported previously (3). An attempt was made to identify individual seminiferous tubules that were larger, more opaque and whiter than other tubules in the testicular parenchyma, which were considered to likely contain spermatozoa. If all tubules were seen to have an identical morphological appearance, at least three samples (upper, middle, and lower) were obtained. If no sperm were obtained from the initial sample, the same procedure was performed in the contralateral testis (in 20\% of the cases). At the same time of testicular intervention in both procedures, a small tissue specimen was placed in Bouin's solution and sent for histopathological examination.

Data collection was carried out following the principles outlined in the Declaration of Helsinki; after approval of the IRCCS Fondazione Ca' Granda Ospedale Maggiore Policlinico Ethical Committee, all patients signed an informed consent agreeing to supply their own anonymous data for this and future studies.

Data are presented as means (SD; ranges). The statistical significance of differences in means and proportions was tested with the one-way analysis of variance (ANOVA) and Pearson chi-square test, respectively. A 95\% confidence interval was estimated for the association of categorical 
parameters. Exploratory analyses were initially applied to all variables; variables were retained for analysis when deemed clinically significant to the results. Descriptive statistics tested the association between clinical and hormonal variables and the sperm retrieval rate according to the surgical technique. Logistic regression univariable analysis (UVA) and multivariable analysis (MVA) tested the associa-

Table 1.

Characteristics and descriptive statistics of patients (No. $=145$ ).

\begin{tabular}{|c|c|c|c|c|}
\hline & Overall & cTESE & mTESE & $P$ value $(F)^{*}$ \\
\hline No. of patients [No. (\%)] & $145(100)$ & $96(66.2)$ & $49(33.8)$ & \\
\hline Age (years) & & & & $0.55(0.34)$ \\
\hline Mean (SD) & $35.4(5.4)$ & $35.6(5.4)$ & $34.9(5.5)$ & \\
\hline Range & $21-54$ & $21-54$ & $23-48$ & \\
\hline Categorized age [No. (\%)] & & & & $0.82\left(x^{2}, 0.9\right)$ \\
\hline $50-60$ & $3(2.1)$ & $2(2.6)$ & $1(2.9)$ & \\
\hline $61-70$ & $73(50.3)$ & $48(50.0)$ & $25(50.0)$ & \\
\hline $71-80$ & 67 (46.2) & $44(44.9)$ & $23(47.1)$ & \\
\hline$\geq 81$ & $2(1.4)$ & $2(2.6)$ & $0(0.0)$ & \\
\hline BMI $\left(\mathrm{kg} / \mathrm{m}^{2}\right)$ & & & & $0.07(4.09)$ \\
\hline Mean (SD) & $26.8(3.4)$ & $26.2(3.1)$ & $29.0(3.7)$ & \\
\hline Range & $19.0-34.9$ & $19.0-31.1$ & 24.1-34.9 & \\
\hline 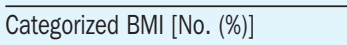 & & & & $0.36\left(x^{2}, 2.00\right)$ \\
\hline $18.5-24.9$ & $39(26.8)$ & 32 (33.3) & $7(14.3)$ & \\
\hline $25-29.9$ & 83 (57.2) & 55 (57.1) & 28 (57.1) & \\
\hline$\geq 30$ & $23(15.8)$ & $9(9.5)$ & $14(28.6)$ & \\
\hline$\overline{\mathrm{CCl}[\text { [No. }(\%)]}$ & & & & $0.74\left(x^{2}, 0.11\right)$ \\
\hline $\mathrm{CCl} O$ & $110(75.8)$ & 75 (77.8) & $35(72.7)$ & \\
\hline $\mathrm{CCl} \geq 1$ & $35(24.1)$ & $21(22.2)$ & $14(27.3)$ & \\
\hline Current Smokers [No. (\%)] & $35(24.1)$ & $21(22.2)$ & $14(27.3)$ & $0.72\left(x^{2}, 0.13\right)$ \\
\hline $\begin{array}{l}\text { Varicocele [No. (\%)] } \\
\text { (N) }\end{array}$ & $52(35.8)$ & $35(36.5)$ & $17(34.6)$ & $0.86\left(x^{2}, 0.31\right)$ \\
\hline Cryptorchidism [No. (\%)] & $27(18.6)$ & $22(23.2)$ & $5(9.1)$ & $0.15\left(x^{2}, 2.1\right)$ \\
\hline Positive SRR [No. (\%)] & $64(44.1)$ & $40(41.7)$ & $24(49.0)$ & $0.40\left(x^{2}, 0.70\right)$ \\
\hline Histologic reports [No. (\%)] & & & & $0.30\left(x^{2}, 2.39\right)$ \\
\hline Hypospermatogenesis & $49(33.7)$ & $28(28.9)$ & $21(42.6)$ & \\
\hline Maturation arrest & $32(22.0)$ & $23(23.7)$ & $9(19.1)$ & \\
\hline Sertoli cell-only syndrome & $64(44.1)$ & $45(47.4)$ & $19(38.3)$ & \\
\hline Postop. complications [No. (\%)] & $3(2.0)$ & $2(2.1)$ & $1(2.0)$ & $0.43\left(x^{2}, 1.04\right)$ \\
\hline
\end{tabular}

Table 2.

Hormonal characteristics of patients (No. $=145$ ).

\begin{tabular}{|c|c|c|c|c|}
\hline & Overall & cTESE & mTESE & $P$ value $(\mathbf{F})^{*}$ \\
\hline$\overline{\mathrm{FSH}}(\mathrm{mUl} / \mathrm{mL})$ & & & & $0.04(8.89)$ \\
\hline Mean (SD) & $14.8(11.8)$ & $12.8(11.1)$ & $20.9(12.1)$ & \\
\hline Range & $1.71-59$ & $1.71-52.3$ & $1.81-59$ & \\
\hline $\mathrm{LH}(\mathrm{mUl} / \mathrm{mL})$ & & & & $0.15(2.12)$ \\
\hline Mean (SD) & $5.7(4.3)$ & $5.3(4.1)$ & $7.2(4.9)$ & \\
\hline Range & $1.91-18.5$ & $1.91-18.5$ & $2.55-17.7$ & \\
\hline $\mathrm{tT}(\mathrm{ng} / \mathrm{mL})$ & & & & $0.12(2.36)$ \\
\hline Mean (SD) & $5.0(4.1)$ & $5.4(4.5)$ & $3.6(1.7)$ & \\
\hline Range & 1.17-24.1 & $1.17-24.1$ & $2.16-7.58$ & \\
\hline$\overline{\mathrm{tT}}<3 \mathrm{ng} / \mathrm{mL}[$ No. $(\%)]$ & $34(23.4)$ & $18(19.2)$ & $16(33.3)$ & $0.25\left(x^{2}, 1.33\right)$ \\
\hline$\overline{\mathrm{PRL}}(\mathrm{ng} / \mathrm{mL})$ & & & & $0.54(0.36)$ \\
\hline Mean (SD) & $26.3(9.2)$ & $30.1(10.6)$ & $11.7(6.44)$ & \\
\hline Range & 4.6-68.1 & $4.6-68.1$ & $5.0-26$ & \\
\hline
\end{tabular}

tions between clinical and laboratory predictors and sperm retrieval failure. Moreover, logistic regression analyses tested the association between clinical predictors (e.g age, FSH, testicular histology and surgical technique) and positive sperm retrieval in patients older than the median value of 35 years and in those with FSH $>1.5 \mathrm{~N}$ (18 mUI/mL). Statistical tests were performed using SPSS v. 19 (IBM Corp., Armonk, NY, USA).

All tests were two sided, with a significance level set at 0.05 .

\section{RESULTS}

Table 1 lists the characteristics and the descriptive statistics of the entire cohort of individuals. Overall, 96 (66.2\%) and 49 (33.8\%) infertile patients were submitted to cTESE and MTESE, respectively. The two groups did not differ in terms of age, BMI and CCI. There were no differences in terms of lifestyle factors, history of cryptorchidism, presence of varicocele and postoperative complications between groups. Histologic reports showed hypospermatogenesis, maturation arrest and Sertoli cell-only syndrome in 49 (33.7\%), 32 (22\%) and $64(44.1 \%)$ patients, respectively, with no difference according to the surgical technique. The success rate of sperm retrieval in patients with NOA was comparable between mTESE and cTESE (49.0\% vs. $41.7 \%, \mathrm{p}=0.40$ ).

Table 2 depicts the hormonal characteristics of infertile patients according to the surgical technique. No differences were found between groups in terms of hormonal parameters with the exception of FSH values that were higher in mTESE patients (20.9 vs. $12.8 \mathrm{mUI} / \mathrm{ml}$; $\mathrm{p}=0.004$ ).

Table 3 shows the influence of clinical parameters (age, FSH values and histological reports) on the success rate of sperm retrieval between groups. Sperm retrieval rate was positive in 22/61 (36.1\%) patients with $\mathrm{FSH} \geq 1.5 \mathrm{~N}$ (18 $\mathrm{mIU} / \mathrm{mL}$ ) (18/30 with mTESE, 4/31 with cTESE) and in 42/84 (50.0\%) patients with $\mathrm{FSH}<1.5 \mathrm{~N}$ (6/19 with mTESE, 36/65 with cTESE). mTESE resulted in a higher SRR for those with FSH $\geq 1.5 \mathrm{~N}$ $(p=0.001)$. Age had a significant impact on sperm retrieval rate in the two groups. In patients younger than 35 years, SRR was positive in $48.9 \%$ and $41.6 \%$ of men submitted to cTESE and mTESE, respectively. However, for those older than 35 years, mTESE resulted in a significantly higher SRR $(56.0 \%$ vs. $35.0 \%, p=0.03)$. The influence of histological diagnosis on the success rate of 
Table 3.

Sperm retrieval rate according to the class of FSH values, age and histologic reports.

\begin{tabular}{|lccc|}
\hline & cTESE & mTESE & P value (F)* \\
\hline FSH $(\mathrm{mUl} / \mathrm{mL})$ & $36 / 65(55.3 \%)$ & $6 / 19(31.5 \%)$ & $0.66(0.18)$ \\
$<18$ & $4 / 31(12.9 \%)$ & $18 / 30(60.0 \%)$ & $0.001(7.0)$ \\
$>18$ & $23 / 47(48.9 \%)$ & $10 / 24(41.6 \%)$ & $0.67(0.18)$ \\
\hline Age (years) & $17 / 49(35.0 \%)$ & $14 / 25(56.0 \%)$ & $0.03(4.27)$ \\
$\quad<35$ & $18 / 28(64.2 \%)$ & $12 / 21(55.0 \%)$ & $0.77(0.01)$ \\
$>35$ & $8 / 23(34.7 \%)$ & $3 / 9(33.3 \%)$ & $0.76(0.09)$ \\
\hline Histologic reports & $14 / 45(31.1 \%)$ & $9 / 19(50.0 \%)$ & $0.03(4.29)$ \\
$\quad$ Hypospermatogenesis & & & \\
$\quad$ Maturation arrest & & & \\
Sertoli cell-only syndrome & &
\end{tabular}

Table 4.

Logistic regression models predicting positive sperm retrieval (OR; $p$ value $[95 \% \mathrm{Cl}]$ ) in patients with age $>35$ years and $\mathrm{FSH}>18 \mathrm{mUl} / \mathrm{mL}$.

\begin{tabular}{|c|c|c|c|c|}
\hline & \multicolumn{4}{|c|}{ Positive sperm retrieval } \\
\hline & \multicolumn{2}{|c|}{ Age $>35$ years } & \multicolumn{2}{|c|}{$\mathrm{FSH}>18 \mathrm{mUl} / \mathrm{mL}$} \\
\hline & UVA model & MVA model & UVA model & MVA model \\
\hline cTESE vs. mTESE & $\begin{array}{l}13.40 ; 0.04 \\
(1.41-21.17)\end{array}$ & $\begin{array}{c}7.22 ; 0.002 \\
(2.01-15.82)\end{array}$ & $\begin{array}{l}12.66 ; 0.001 \\
(2.49-24.22)\end{array}$ & $\begin{array}{c}9.52 ; 0.018 \\
(1.96-19.26)\end{array}$ \\
\hline Age & - & - & $\begin{array}{c}0.23 ; 0.76 \\
(0.11-1.15)\end{array}$ & $\begin{array}{c}0.11 ; 0.18 \\
(0.06-1.89)\end{array}$ \\
\hline$\overline{\mathrm{FSH}}$ & $\begin{array}{c}0.97 ; 0.10 \\
(0.88-1.02)\end{array}$ & $\begin{array}{l}0.18 ; 0.90 \\
(0.05-1.17)\end{array}$ & - & - \\
\hline \multicolumn{5}{|l|}{ Histologic report } \\
\hline Hypospermatogenesis & Ref. & Ref. & Ref. & Ref. \\
\hline Sertoli cell-only syndrome & $\begin{array}{c}0.21 ; 0.12 \\
(0.02-1.52)\end{array}$ & $\begin{array}{l}0.12 ; 0.24 \\
(0.03-4.31)\end{array}$ & $\begin{array}{c}0.20 ; 0.11 \\
(0.03-1.43)\end{array}$ & $\begin{array}{l}0.31 ; 0.78 \\
(0.21-1.63)\end{array}$ \\
\hline Maturation arrest & $\begin{array}{c}0.34 ; 0.19 \\
(0.06-1.74)\end{array}$ & $\begin{array}{c}0.11 ; 0.21 \\
(0.05-3.40)\end{array}$ & $\begin{array}{c}0.23 ; 0.11 \\
(0.04-1.35)\end{array}$ & $\begin{array}{c}0.34 ; 0.78 \\
(0.20-1.45)\end{array}$ \\
\hline
\end{tabular}

Table 5.

Logistic regression models predicting negative sperm retrieval (OR; $p$ value $[95 \% \mathrm{Cl}]$ ) in the whole cohort $(n=145)$.

\begin{tabular}{|c|c|c|c|c|}
\hline & \multicolumn{4}{|c|}{ Sperm retrieval failure } \\
\hline & \multicolumn{2}{|l|}{ UVA model } & \multicolumn{2}{|l|}{ MVA model } \\
\hline & OR (95\% Cl) & $P$ value & OR (95\% Cl) & $P$ value \\
\hline$\overline{\text { Age }}$ & $1.04(0.95-1.13)$ & 0.38 & $1.13(0.83-1.55)$ & 0.41 \\
\hline$\overline{\mathrm{FSH}}$ & $1.11(1.02-1.16)$ & 0.008 & $1.31(1.01-1.68)$ & 0.03 \\
\hline $\mathrm{LH}$ & $0.76(0.91-1.21)$ & 0.46 & $0.65(0.89-1.26)$ & 0.07 \\
\hline Testosterone & $0.96(0.92-1.22)$ & 0.40 & $0.98(0.79-1.21)$ & 0.87 \\
\hline \multicolumn{5}{|l|}{ Histologic report } \\
\hline Hypospermatogenesis & Ref. & & Ref. & \\
\hline Sertoli cell-only syndrome & $4.21(1.31-3.32)$ & 0.014 & $2.17(1.01-8.87)$ & 0.01 \\
\hline Maturation arrest & $3.20(1.16-1.32)$ & 0.04 & $1.87(1.02-7.52)$ & 0.04 \\
\hline
\end{tabular}

association between mTESE and positive sperm retrieval in older men and in those with higher FSH levels we conducted a logistic regression analysis assessing the relationship between clinical predictors (e.g age, FSH, testicular histology and surgical technique) and positive sperm retrieval in the two groups (Table 4). In patients older than the median value of 35 years mTESE was univariably associated with positive SR ( $p=0.04)$ and emerged as the only independent predictor of positive SR (OR 7.22; p = 0.002) after adjusting for FSH values and testicular histology. Similarly, in men with FSH $>18 \mathrm{mUI} / \mathrm{mL}$, mTESE was associated with positive SRR ( $p=0.001)$ at UVA and showed independent predictor status for positive SR (OR 9.52; $\mathrm{p}=0.018$ ) at MVA, after adjusting for age and testicular histology.

Table 5 details UVA and MVA logistic regression models testing the associations between predictors and sperm retrieval failure. UVA showed that higher FSH ( $p=0.008)$, a histological report of SCOS ( $p=0.014)$ and MA ( $p=0.04)$ were associated with negative SRR. Conversely, age, LH and testosterone values were not. Similarly, logistic MVA revealed that FSH levels (OR 1.3, p = 0.03), histological reports of SCOS (OR 2.17, $\mathrm{p}=0.01$ ) and MA (OR 1.87, $\mathrm{p}=$ 0.04) achieved independent predictor status for sperm retrieval failure.

\section{Discussion}

This study was conducted to evaluate potential differences in terms of SRRs between $\mathrm{mTESE}$ and cTESE in our cohort of NOA men, and to determine which patients could most benefit from each procedure. We found that mTESE yields overall greater, albeit not significantly, rates of sperm retrieval than cTESE. Secondly, we noted that mTESE lead to a higher SRR than cTESE in older patients ( $>35$ years), in patients with elevated FSH (i.e > $18 \mathrm{mUI} / \mathrm{ml}$ ) and in those with a histological diagnosis of SCOS. mTESE emerged as independent predictor of positive SR in patients older than 35 years and in those with FSH >18 mUI/mL.

sperm retrieval was also considered. We obtained spermatozoa via cTESE in $64.2 \%$ and via mTESE in $55.0 \%$ of men with a histological diagnosis of hypospermatogenesis. In case of MA, we retrieved sperm in $34.7 \%$ of patients via cTESE and in $33.3 \%$ via mTESE. For those with SCOS, mTESE produced a significantly higher SRR (50.0\% vs. $31.1 \%$ with cTESE, $\mathrm{p}=0.03$ ). In order to strengthen the
Moreover, the most reliable predictors of negative sperm retrieval were elevated FSH and both SCOS and MA histology.

This study was prompted by existing controversies in the scientific literature regarding the role of mTESE and cTESE as treatment options for azoospermic men. In fact, determining which of the two procedures is more 
likely to produce a better outcome remains challenging since, to date, a general consensus is still lacking among the different authors.

In terms of SRR, the current literature reports quite unanimously a superiority of mTESE over conventional TESE (4$6,9,14)$. For instance, Okada et al. (4) reported a SRR of $16.7 \%$ for the cTESE group and $44.6 \%$ for mTESE. More recently Ghalayini et al. (6) found that sperm retrieval was successful in 56.9\% patients undergoing mTESE in comparison with only $38.2 \%$ of cTESE patients. The present study showed a slight, but not statistically significant, superiority of mTESE over cTESE in terms of SRR $(49.0 \%$ vs $41.7 \%$ ). Other authors have also shown a superiority of mTESE over cTESE in terms of overall SRR but without a statistically significant difference between groups (15).

Previous studies found conventional TESE to be associated with significantly higher complication rates than mTESE $(4,7)$. Acute and chronic ultrasonographic abnormalities such as haematoma, loss of testicular tissue, inflammatory changes and permanent devascularisation may be found after cTESE (7). Moreover, cTESE may be associated with higher rates of wound infection and iatrogenic hypogonadism that mTESE (4). We did not find any differences between groups in terms of postoperative complications, probably due to the low rate of adverse events in our cohort (3 patients, 2 with heamtomas and 1 with wound infections).

Conventional TESE could still offer some advantages over mTESE. Firstly, microsurgical training generally represents a lengthy, expensive and demanding process that likely cannot be sustained in every institution. In fact, Ishikawa et al. (15) showed a steeper learning curve for mTESE compared with cTESE, highlighting a positive correlation between surgical outcomes and turnover of mTESE operations. Secondly, mTESE requires dedicated and expensive equipment (especially an operating microscope) as well as longer operative times. It is therefore difficult to find a compromise that guarantees the best quality at the lowest cost. One way to deal with this issue could be tailor patient treatment based on robust predictive factors for positive SRR. To date, many promising candidates for predictors have been proposed, but a general consensus is still lacking.

Testicular volume is amongst the frequently proposed predictors. However, Colpi et al. (14) and Ghalayini et al. (6) found contrasting results, with the former showing no correlation and the latter a positive correlation between higher testicular volume and SRRs.

Another factor, advanced age, is generally associated with poorer sperm parameters and reproductive outcomes (16). However, recent studies have shown that age may not adversely affect sperm retrieval in men undergoing TESE $(10,17)$. Of clinical importance, we found significantly higher SRRs with mTESE in patients older than the median value of 35 years when compared to aged-match patients undergoing CTESE. Moreover, mTESE was an independent predictor of positive SR in men older than 35 years after adjusting for FSH values and testicular histology. This finding could provide an easy to obtain and reliable predictor for identifying the best candidates to submit to mTESE, especially if they belong to poor prognosis groups.
Serum hormonal values represent another potentially useful predictor of SRRs. Colpi et al. (14) and Ghalayini et al. (6) reported a higher failure in sperm retrieval among patients with increased FSH levels. Conversely, Ramasamy et al. (18) observed comparable or better results in men with high FSH in terms of microsurgical retrieval. Our study strongly supports the negative association between SRR and FSH levels as we found FSH to be an independent predictor of SR failure in the overall cohort of patients and, more importantly, we showed that SRR was significantly higher in mTESE patients with high FSH when compared with cTESE patients with comparable hormonal values. To strengthen this finding we performed a multivariable analysis to predict positive SRR in men with higher FSH values and we found that mTESE was independent predictor of positive SR after adjusting for age and testicular histology.

Testicular histological pattern is one of the most promising parameters for providing both the clinician and patient with a reliable and robust predictive tool for SR outcomes. Unfortunately, and differently from the other aforementioned factors, it can be provided only through an invasive surgical procedure and is, therefore, mainly evaluated intraoperatively or retrospectively.

Our data showed that SCOS patients had significantly higher SRRs from mTESE compared to cTESE. Moreover, SCOS and MA were independent predictors of SR failure. Our outcomes are consistent with previous studies showing the superiority of mTESE over cTESE in SR outcomes for SCOS men $(4,6)$.

Similarly, the significant association between both SCOS and MA and sperm retrieval failure has been previously reported $(6,19)$. It can be concluded that progressively worse histological patterns are associated with lower retrieval results. We can therefore speculate that improvements in SRR in poor prognosis patients (those with older age, high FSH values and SCOS) could be achieved performing mTESE instead of cTESE.

Our study presents some limitations. First, it is not a randomized controlled study; our patients were assigned to one of the two groups on the basis of the operating theatre waiting list. Second, we didn't consider some variables such as inhibin B, Johnsen score and testicular volume, which have been shown to have a relevant albeit controversial predictive value. Finally, we were unable evaluate the pregnancy rate associated with ICSI procedures following sperm retrievals. Clinical pregnancy followed by the delivery of a healthy child is the main goal of all the procedures described, but it requires extensive follow up. Nevertheless, our findings offer some positive implications that could be useful to clinicians not only to offer realistic counselling to couples undergoing ARTs treatments but also to tailor the best treatment to the patient who could most benefit from it. For example, we showed that mTESE was associated with a better SRR than cTESE in older patients. This finding is important in light of the fact that advanced age is associated with decreasing testosterone levels and mTESE guarantees better preservation of the testicular tissue. Thus, with mTESE representing a reduced risk of hypogonadism, the higher sperm retrieval rate would make mTESE doubly beneficial for the patient. Secondly, we confirmed the high predictive value of histology, espe- 
cially for cases of poor prognosis (SCOS and MA), with mTESE yielding the best results for SCOS patients. A preoperative histological diagnosis is however difficult to obtain unless the patient has already undergone a sperm retrieval procedure. A proposed alternative could be a stepwise approach, such as that described by Franco et al. (20), which gradually increases surgical complexity only when necessary. It is undeniable, however, that patients with poor prognosis, as defined by histology, would greatly benefit from moving directly to a microsurgical approach and avoiding procedures that are extremely unlikely to produce positive results.

\section{Conclusions}

In conclusion, microdissection and conventional TESE showed comparable success rates for sperm retrieval in our cohort of patients with NOA. mTESE seems to be beneficial for patients older than 35 years, in patients with high FSH values, or when SCOS can be predicted. Given the high costs associated with the mTESE approach, the identification of candidates most likely to benefit from this procedure is a major clinical need.

\section{REFERENCES}

1. Su LM, Palermo GD, Goldstein M, et al. Testicular sperm extraction with intracytoplasmic sperm injection for nonobstructive azoospermia: testicular histology can predict success of sperm retrieval. J Urol. 1999; 161:112-116.

2. Kahraman S, Ozgur S, Alatas C, et al. High implantation and pregnancy rates with testicular sperm extraction and intracytoplasmic sperm injection in obstructive and non-obstructive azoospermia. Hum Reprod. 1996; 11:673-676.

3. Schlegel PN. Testicular sperm extraction: microdissection improves sperm yield with minimal tissue excision. Hum Reprod. 1999; 14:131-5.

4. Okada H, Dobashi M, Yamazaki T, et al. Conventional versus microdissection testicular sperm extraction for nonobstructive azoospermia. J Urol. 2002; 168:1063-7.

5. Ramasamy R, Yagan N, Schlegel PN. Structural and functional changes to the testis after conventional versus microdissection testicular sperm extraction. Urology 2005; 65:1190-4.

6. Ghalayini IF, Al-Ghazo MA, Hani OB, et al. Clinical Comparison of Conventional Testicular Sperm Extraction and Microdissection Techniques for Non-Obstructive Azoospermia. J Clin Med Res. 2011; 3:124- 131

7. Schlegel PN, Su LM. Physiological consequences of testicular sperm extraction. Hum Reprod 1997; 12:1688-92.

8. Silber SJ. Microsurgical TESE and the distribution of spermatogenesis in non-obstructive azoospermia. Hum Reprod. 2000; 15:2278-84.

9. Tsujimura A, Matsumiya K, Miyagawa Y, et al. Conventional multiple or microdissection testicular sperm extraction: a comparative study. Hum Reprod. 2002; 17:2924-9.

10. Saccà A, Pastore AL, Roscigno M, et al. Conventional testicular sperm extraction (TESE) and non-obstructive azoospermia: is there still a chance in the era of microdissection TESE? Results from a single non-academic community hospital. Andrology. 2016; 4:425-9.

11. World Health Organization web chapter on couple's infertility. http://www.who.int/reproductivehealth/topics/infertility/definitions (Accessed September 3rd, 2015).
12. Charlson ME, Pompei P, Ales KL, MacKenzie CR. A new method of classifying prognostic comorbidity in longitudinal studies: development and validation. J Chronic Dis. 1987; 40:373-83.

13. Bhasin S, Cunningham GR, Hayes FJ, et al. Testosterone Therapy in men with androgen deficieny syndromes: an American Society clinical practice guidelines. J Clin Endocrinol Metab. 2010; 95:2536-59.

14. Colpi GM, Colpi EM, Piediferro G, et al. Microsurgical TESE versus conventional TESE for ICSI in non-obstructive azoospermia: a randomized controlled study. Reprod Biomed Online. 2009; 18:315-9.

15. Ishikawa $T$, Nose R, Yamaguchi $K$, et al. Learning curves of microdissection testicular sperm extraction for nonobstructive azoospermia. Fertil Steril. 2010; 94:1008-11.

16. Grunewald S, Glander HJ, Paasch U, Kratzsch J. Age-dependent inhibin $B$ concentration in relation to FSH and semen sample qualities: a study in 2448 men. Reproduction 2013; 145:237-244.

17. Ramasamy R, Trivedi NN, Reifsnyder JE, et al. Age does not adversely affect sperm retrieval in men undergoing microdissection testicular sperm extraction. Fertil Steril. 2014; 101: 653-5.

18. Ramasamy R, Lin K, Gosden LV, et al. High serum FSH levels in men with nonobstructive azoo spermia does not affect success of microdissection testicular sperm extraction. Fertil Steril. 2009; 92:590-3.

19. Caroppo E, Colpi EM, Gazzano G, et al. Testicular histology may predict the successful sperm retrieval in patients with nonobstructive azoospermia undergoing conventional TESE: a diagnostic accuracy study. J Assist Reprod Genet. 2017; 34:149-154.

20. Franco G, Scarselli F, Casciani V, et al. A novel stepwise microTESE approach in non obstructive azoospermia. BMC Urol. 2016; $16,20$.

\section{Correspondence}

Elia Maglia, MD

Elia.maglia@studenti.unimi.it

Luca Boeri, MD (Corresponding Author)

Dr.lucaboeri@gmail.com

Matteo Fontana, MD

Teo.fontana@me.com

Andrea Gallioli, MD

Andrea.gallioli@gmail.com

Elisa De Lorenzis, MD

Elisa.delorenzis@gmail.com

Franco Palmisano, MD

Franco.palmisano@hotmail.it

Stefano Paolo Zanetti, MD

Stefano.p.zanetti@gmail.com

Gianluca Sampogna, MD

Gianluca.sampogna@unimi.it

Mariapia Serrago, MD

Mariapia.serrago@policlinico.mi.it

Franco Gadda, MD

Franco.gadda@policlinico.mi.it

Emanuele Montanari, MD

IEmanuele.montanari@unimi.it

RCCS Fondazione Ca' Granda, Ospedale Maggiore Policlinico,

Department of Urology

via della Commenda 15, 20122 Milano, Italy

Liliana Restelli, MD

Liliana.restelli@policlinico.mi.it

Edgardo Somigliana, MD

Edgardo.somigliana@policlinico.mi.it

IRCCS Fondazione Ca' Granda, Ospedale Maggiore Policlinico,

U.O.S.D Procreazione Medicalmente Assistita

via Festa del Perdono 12, 20122 Milano, Italy 